\title{
An example of noncommutative deformations
}

\author{
Eivind Eriksen \\ Oslo University College \\ Postboks 4, St. Olavs plass \\ N-0130 Oslo, Norway \\ E-mail: eeriksen@hio.no
}

August 23, 2021

\begin{abstract}
We compute the noncommutative deformations of a family of modules over the first Weyl algebra. This example shows some important properties of noncommutative deformation theory that separates it from commutative deformation theory.
\end{abstract}

MSC2000: 14D15; 13D10

\section{Introduction}

Let $k$ be an algebraically closed field and let $A$ be an associative $k$-algebra. For any left $A$-module $M$, there is a flat commutative deformation functor

$$
\operatorname{Def}_{M}: I \rightarrow \text { Sets }
$$

defined on the category I of local Artinan commutative $k$-algebras with residue field $k$. We recall that for an object $R \in \mathrm{I}$, a flat deformation of $M$ over $R$ is a pair $\left(M_{R}, \tau\right)$, where $M_{R}$ is an $A-R$ bimodule (on which $k$ acts centrally) that is $R$-flat, and $\tau: M_{R} \otimes_{R} k \rightarrow M$ is an isomorphism of left $A$-modules. Moreover, $\left(M_{R}, \tau\right) \sim\left(M_{R}^{\prime}, \tau^{\prime}\right)$ as deformations in $\operatorname{Def}_{M}(R)$ if there is an isomorphism $\eta: M_{R} \rightarrow M_{R}^{\prime}$ of $A$ - $R$ bimodules such that $\tau=\tau^{\prime} \circ(\eta \otimes 1)$.

Laudal introduced noncommutative deformations of modules in Laudal [2]. For any finite family $\mathcal{M}=\left\{M_{1}, \ldots, M_{p}\right\}$ of left $A$-modules, there is a noncommutative deformation functor

$$
\operatorname{Def}_{\mathcal{M}}: \mathrm{a}_{p} \rightarrow \text { Sets }
$$

defined on the category $\mathrm{a}_{p}$ of $p$-pointed Artinian $k$-algebras. We recall that an object $R$ of $\mathrm{a}_{p}$ is an Artinian ring $R$, together with a pair of structural ring homomorphisms $f: k^{p} \rightarrow R$ and $g: R \rightarrow k^{p}$, such that $g \circ f=\mathrm{id}$ and the radical $I(R)=\operatorname{ker}(g)$ is nilpotent. The morphisms of $\mathrm{a}_{p}$ are ring homomorphisms that commute with the structural morphisms.

A deformation of the family $\mathcal{M}$ over $R$ is a $(p+1)$-tuple $\left(M_{R}, \tau_{1}, \ldots, \tau_{p}\right)$, where $M_{R}$ is an $A$ - $R$ bimodule (on which $k$ acts centrally) such that $M_{R} \cong\left(M_{i} \otimes_{k} R_{i j}\right)$ as right $R$-modules, and $\tau_{i}: M_{R} \otimes_{R} k_{i} \rightarrow M_{i}$ is an isomorphism of left $A$-modules for $1 \leq i \leq p$. By definition,

$$
\left(M_{i} \otimes_{k} R_{i j}\right)=\underset{1 \leq i, j \leq p}{\oplus} M_{i} \otimes_{k} R_{i j}
$$

with the natural right $R$-module structure, and $k_{1}, \ldots, k_{p}$ are the simple left $R$-modules of dimension one over $k$. Moreover, $\left(M_{R}, \tau_{1}, \ldots, \tau_{p}\right) \sim\left(M_{R}^{\prime}, \tau_{1}^{\prime}, \ldots, \tau_{p}^{\prime}\right)$ as deformations in $\operatorname{Def}_{\mathcal{M}}(R)$ if there is an isomorphism $\eta: M_{R} \rightarrow M_{R}^{\prime}$ of $A$ - $R$ bimodules such that $\tau_{i}=\tau_{i}^{\prime} \circ(\eta \otimes 1)$ for $1 \leq i \leq p$. 
There is a cohomology theory and an obstruction calculus for Def $\mathcal{M}_{\mathcal{M}}$, see Laudal [2] and Eriksen [1]. We compute the noncommutative deformations of a family $\mathcal{M}=\left\{M_{1}, M_{2}\right\}$ of modules over the first Weyl algebra using the constructive methods described in Eriksen [1].

\section{An example of noncommutative deformations of a family}

Let $k$ be an algebraically closed field of characteristic 0 , let $A=k[t]$, and let $D=\operatorname{Diff}(A)$ be the first Weyl algebra over $k$. We recall that $D=k\langle t, \partial\rangle /(\partial t-t \partial-1)$. Let us consider the family $\mathcal{M}=\left\{M_{1}, M_{2}\right\}$ of left $D$-modules, where $M_{1}=D / D \cdot \partial \cong A$ and $M_{2}=D / D \cdot t \cong k[\partial]$. We shall compute the noncommutative deformations of the family $\mathcal{M}$.

In this example, we use the methods described in Eriksen [1] to compute noncommutative deformations. In particular, we use the cohomology $\mathrm{YH}^{n}\left(M_{j}, M_{i}\right)$ of the Yoneda complex

$$
Y C^{p}\left(M_{j}, M_{i}\right)=\prod_{m \geq 0} \operatorname{Hom}_{D}\left(L_{m, j}, L_{m-p, i}\right)
$$

for $1 \leq i, j \leq 2$, where $\left(L_{*, i}, d_{*, i}\right)$ is a free resolution of $M_{i}$, and an obstruction calculus based on these free resolutions. We recall that $\mathrm{YH}^{n}\left(M_{j}, M_{i}\right) \cong \operatorname{Ext}_{D}^{n}\left(M_{j}, M_{i}\right)$.

Let us compute the cohomology $\mathrm{YH}^{n}\left(M_{j}, M_{i}\right)$ for $n=1,2,1 \leq i, j \leq 2$. We use the free resolutions of $M_{1}$ and $M_{2}$ as left $D$-modules given by

$$
\begin{aligned}
& 0 \leftarrow M_{1} \leftarrow D \stackrel{\cdot \partial}{\leftarrow} D \leftarrow 0 \\
& 0 \leftarrow M_{2} \leftarrow D \stackrel{\cdot t}{\leftarrow} D \leftarrow 0
\end{aligned}
$$

and the definition of the differentials $Y C^{0}\left(M_{j}, M_{i}\right) \rightarrow Y C^{1}\left(M_{j}, M_{i}\right) \rightarrow Y C^{2}\left(M_{j}, M_{i}\right)=0$ in the Yoneda complex, and obtain

$$
\begin{array}{ll}
\mathrm{YH}^{1}\left(M_{1}, M_{1}\right) \cong \operatorname{Ext}_{D}^{1}\left(M_{1}, M_{1}\right)=0 & \mathrm{YH}^{1}\left(M_{1}, M_{2}\right) \cong \operatorname{Ext}_{D}^{1}\left(M_{1}, M_{2}\right)=k \cdot \xi_{21} \\
\mathrm{YH}^{1}\left(M_{2}, M_{1}\right) \cong \operatorname{Ext}_{D}^{1}\left(M_{2}, M_{1}\right)=k \cdot \xi_{12} & \mathrm{YH}^{1}\left(M_{2}, M_{2}\right) \cong \operatorname{Ext}_{D}^{1}\left(M_{2}, M_{2}\right)=0
\end{array}
$$

The base vector $\xi_{i j}$ is represented by the 1-cocycle given by $D \stackrel{\cdot 1}{\rightarrow} D$ in $Y C^{1}\left(M_{j}, M_{i}\right)$ when $i \neq j$. Since $Y C^{2}\left(M_{j}, M_{i}\right)=0$ for all $i, j$, it is clear that $\mathrm{YH}^{2}\left(M_{j}, M_{i}\right) \cong \operatorname{Ext}_{D}^{2}\left(M_{j}, M_{i}\right)=0$ for $1 \leq i, j \leq 2$.

We conclude that $\operatorname{Def}_{\mathcal{M}}$ is unobstructed. Hence, in the notation of Eriksen [1, the prorepresenting hull $H$ of $\operatorname{Def}_{\mathcal{M}}$ is given by

$$
H=\left(\begin{array}{ll}
H_{11} & H_{12} \\
H_{21} & H_{22}
\end{array}\right) \cong\left(\begin{array}{cc}
k\left[\left[s_{12} s_{21}\right]\right] & \left\langle s_{12}\right\rangle \\
\left\langle s_{21}\right\rangle & k\left[\left[s_{21} s_{12}\right]\right]
\end{array}\right)
$$

where $\left\langle s_{12}\right\rangle=H_{11} \cdot s_{12} \cdot H_{22}$ and $\left\langle s_{21}\right\rangle=H_{22} \cdot s_{21} \cdot H_{11}$.

In order to describe the versal family $\mathcal{M}_{H}$ of left $D$-modules defined over $H$, we use Mfree resolutions in the notation of Eriksen [1]. In fact, the $D-H$ bimodule $\mathcal{M}_{H}$ has an M-free resolution of the form

$$
0 \leftarrow \mathcal{M}_{H} \leftarrow\left(\begin{array}{ll}
D \widehat{\otimes}_{k} H_{11} & D \widehat{\otimes}_{k} H_{12} \\
D \widehat{\otimes}_{k} H_{21} & D \widehat{\otimes}_{k} H_{22}
\end{array}\right) \stackrel{d^{H}}{\longleftarrow}\left(\begin{array}{cc}
D \widehat{\otimes}_{k} H_{11} & D \widehat{\otimes}_{k} H_{12} \\
D \widehat{\otimes}_{k} H_{21} & D \widehat{\otimes}_{k} H_{22}
\end{array}\right) \leftarrow 0
$$

where $d^{H}=(\cdot \partial) \widehat{\otimes} e_{i}-(\cdot 1) \widehat{\otimes} s_{12}-(\cdot 1) \widehat{\otimes} s_{21}+(\cdot t) \widehat{\otimes} e_{2}$. This means that for any $P, Q \in D$, we have that $d^{H}\left(P \otimes e_{1}\right)=(P \cdot \partial) \widehat{\otimes} e_{1}-(P \cdot 1) \widehat{\otimes} s_{21}$ and $d^{H}\left(Q \otimes e_{2}\right)=(Q \cdot t) \widehat{\otimes} e_{2}-(Q \cdot 1) \widehat{\otimes} s_{12}$. by

We remark that there is a natural algebraization $S$ of the pro-representation hull $H$, given

$$
S=\left(\begin{array}{ll}
S_{11} & S_{12} \\
S_{21} & S_{22}
\end{array}\right) \cong\left(\begin{array}{cc}
k\left[s_{12} s_{21}\right] & \left\langle s_{12}\right\rangle \\
\left\langle s_{21}\right\rangle & k\left[s_{21} s_{12}\right]
\end{array}\right)
$$


In other words, $S$ is an associative $k$-algebra of finite type such that the $J$-adic completion $\widehat{S} \cong H$ for the ideal $J=\left(s_{12}, s_{21}\right) \subseteq S$. The corresponding algebraization $\mathcal{M}_{S}$ of the versal family $\mathcal{M}_{H}$ is given by the $\mathrm{M}$-free resolution

$$
0 \leftarrow \mathcal{M}_{S} \leftarrow\left(\begin{array}{ll}
D \otimes_{k} S_{11} & D \otimes_{k} S_{12} \\
D \otimes_{k} S_{21} & D \otimes_{k} S_{22}
\end{array}\right) \stackrel{d^{S}}{\longleftarrow}\left(\begin{array}{ll}
D \otimes_{k} S_{11} & D \otimes_{k} S_{12} \\
D \otimes_{k} S_{21} & D \otimes_{k} S_{22}
\end{array}\right) \leftarrow 0
$$

with differential $d^{S}=(\cdot \partial) \otimes e_{i}-(\cdot 1) \otimes s_{12}-(\cdot 1) \otimes s_{21}+(\cdot t) \otimes e_{2}$.

We shall determine the $D$-modules parameterized by the family $\mathcal{M}_{S}$ over the noncommutative algebra $S$ - this is much more complicated than in the commutative case. We consider the simple left $S$-modules as the points of the noncommutative algebra $S$, following Laudal [3], 4]. For any simple $S$-module $T$, we obtain a left $D$-module

$$
M_{T}=\mathcal{M}_{S} \otimes_{S} T
$$

Therefore, we consider the problem of classifying simple $S$-modules of dimension $n \geq 1$.

Any $S$-module of dimension $n \geq 1$ is given by a ring homomorphism $\rho: S \rightarrow \operatorname{End}_{k}(T)$, and we may identify $\operatorname{End}_{k}(T) \cong M_{n}(k)$ by choosing a $k$-linear base $\left\{v_{1}, \ldots, v_{n}\right\}$ for $T$. We see that $S$ is generated by $e_{1}, s_{12}, s_{21}$ as a $k$-algebra (since $e_{2}=1-e_{1}$ ), and there are relations

$$
s_{12}^{2}=s_{21}^{2}=0, e_{1}^{2}=e_{1}, e_{1} s_{12}=s_{12}, s_{21} e_{1}=s_{21}, s_{12} e_{1}=e_{1} s_{21}=0
$$

Any $S$-module of dimension $n$ is therefore given by matrices $E_{1}, S_{12}, S_{21} \in M_{n}(k)$ satisfying the matric equations

$$
S_{12}^{2}=S_{21}^{2}=0, E_{1}^{2}=E_{1}, E_{1} S_{12}=S_{12}, S_{21} E_{1}=S_{21}, S_{12} E_{1}=E_{1} S_{21}=0
$$

The $S$-modules represented by $\left(E_{1}, S_{12}, S_{21}\right)$ and $\left(E_{1}^{\prime}, S_{12}^{\prime}, S_{21}^{\prime}\right)$ are isomorphic if and only if there is an invertible matrix $G \in M_{n}(k)$ such that $G E_{1} G^{-1}=E_{1}^{\prime}, G S_{12} G^{-1}=S_{12}^{\prime}, G S_{21} G^{-1}=S_{21}^{\prime}$. Using this characterization, it is a straight-forward but tedious task to classify all $S$-modules of dimension $n$ up to isomorphism for a given integer $n \geq 1$.

Let us first remark that for any $S$-module of dimension $n=1, \rho$ factorizes through the commutativization $k^{2}$ of $S$. It follows that there are exactly two non-isomorphic simple $S$ modules of dimension one, $T_{1,1}$ and $T_{1,2}$, and the corresponding deformations of $\mathcal{M}$ are

$$
M_{1, i}=\mathcal{M}_{S} \otimes_{S} T_{1, i} \cong M_{i} \text { for } i=1,2
$$

This reflects that $M_{1}$ and $M_{2}$ are rigid as left $D$-modules.

We obtain the following list of $S$-modules of dimension $n=2$, up to isomorphism. We have used that, without loss of generality, we may assume that $E_{1}$ has Jordan form:

$$
\begin{array}{rlrl}
E_{1} & =\left(\begin{array}{ll}
0 & 0 \\
0 & 0
\end{array}\right) & S_{12}=\left(\begin{array}{ll}
0 & 0 \\
0 & 0
\end{array}\right) & S_{21}=\left(\begin{array}{ll}
0 & 0 \\
0 & 0
\end{array}\right) \\
E_{1}=\left(\begin{array}{ll}
1 & 0 \\
0 & 1
\end{array}\right) & S_{12}=\left(\begin{array}{ll}
0 & 0 \\
0 & 0
\end{array}\right) & S_{21}=\left(\begin{array}{ll}
0 & 0 \\
0 & 0
\end{array}\right) \\
E_{1}=\left(\begin{array}{ll}
1 & 0 \\
0 & 0
\end{array}\right) & S_{12}=\left(\begin{array}{ll}
0 & 0 \\
0 & 0
\end{array}\right) & S_{21}=\left(\begin{array}{ll}
0 & 0 \\
0 & 0
\end{array}\right) \\
E_{1}=\left(\begin{array}{ll}
1 & 0 \\
0 & 0
\end{array}\right) & S_{12}=\left(\begin{array}{ll}
0 & 0 \\
0 & 0
\end{array}\right) & S_{21}=\left(\begin{array}{ll}
0 & 0 \\
1 & 0
\end{array}\right) \\
E_{1}=\left(\begin{array}{ll}
1 & 0 \\
0 & 0
\end{array}\right) & S_{12}=\left(\begin{array}{ll}
0 & 1 \\
0 & 0
\end{array}\right) & S_{21}=\left(\begin{array}{ll}
0 & 0 \\
0 & 0
\end{array}\right) \\
E_{1}=\left(\begin{array}{ll}
1 & 0 \\
0 & 0
\end{array}\right) & S_{12}=\left(\begin{array}{ll}
0 & 1 \\
0 & 0
\end{array}\right) & S_{21}=\left(\begin{array}{ll}
0 & 0 \\
a & 0
\end{array}\right) \quad \text { for } a \in k^{*}
\end{array}
$$


We shall write $T_{2,1}-T_{2,5}$ and $T_{2,6, a}$ for the corresponding $S$-modules of dimension two. Notice that $T_{2,6, a}$ is simple for all $a \in k^{*}$, while $T_{2,1}-T_{2,5}$ are extensions of simple $S$-modules of dimension one. The corresponding deformations of $\mathcal{M}$ are given by

$$
M_{2,6, a}=\mathcal{M}_{S} \otimes_{S} T_{2,6, a} \quad \text { for } a \in k^{*}
$$

In fact, one may show that $M_{2,6, a} \cong D / D \cdot(t \partial-a)$ for any $a \in k^{*}$. In particular, $M_{2,6, a}$ is a simple $D$-module if $a \notin \mathbb{Z}$, and in this case $M_{2,6, a} \cong M_{2,6, b}$ if and only if $a-b \in \mathbb{Z}$. Furthermore, $M_{2,6,-1} \cong D / D \cdot \partial t, M_{2,6, n} \cong M_{1}$ for $n=1,2, \ldots$, and $M_{2,6,-n} \cong M_{2}$ for $n=2,3, \ldots$.

We obtain the following list of $S$-modules of dimension $n=3$, up to isomorphism. We have used that, without loss of generality, we may assume that $E_{1}$ has Jordan form:

$$
\begin{array}{lll}
E_{1}=\left(\begin{array}{lll}
0 & 0 & 0 \\
0 & 0 & 0 \\
0 & 0 & 0
\end{array}\right) & S_{12}=\left(\begin{array}{ccc}
0 & 0 & 0 \\
0 & 0 & 0 \\
0 & 0 & 0
\end{array}\right) & S_{21}=\left(\begin{array}{lll}
0 & 0 & 0 \\
0 & 0 & 0 \\
0 & 0 & 0
\end{array}\right) \\
E_{1}=\left(\begin{array}{lll}
1 & 0 & 0 \\
0 & 1 & 0 \\
0 & 0 & 1
\end{array}\right) & S_{12}=\left(\begin{array}{lll}
0 & 0 & 0 \\
0 & 0 & 0 \\
0 & 0 & 0
\end{array}\right) & S_{21}=\left(\begin{array}{lll}
0 & 0 & 0 \\
0 & 0 & 0 \\
0 & 0 & 0
\end{array}\right)
\end{array}
$$$$
E_{1}=\left(\begin{array}{lll}
1 & 0 & 0 \\
0 & 1 & 0 \\
0 & 0 & 1
\end{array}\right)
$$$$
S_{21}=\left(\begin{array}{lll}
0 & 0 & 0 \\
0 & 0 & 0 \\
0 & 0 & 0
\end{array}\right)
$$

$$
E_{1}=\left(\begin{array}{lll}
1 & 0 & 0 \\
0 & 0 & 0 \\
0 & 0 & 0
\end{array}\right)
$$$$
S_{12}=\left(\begin{array}{lll}
0 & 0 & 0 \\
0 & 0 & 0 \\
0 & 0 & 0
\end{array}\right)
$$$$
S_{21}=\left(\begin{array}{lll}
0 & 0 & 0 \\
0 & 0 & 0 \\
0 & 0 & 0
\end{array}\right)
$$

$$
E_{1}=\left(\begin{array}{lll}
1 & 0 & 0 \\
0 & 0 & 0 \\
0 & 0 & 0
\end{array}\right)
$$$$
S_{12}=\left(\begin{array}{lll}
0 & 0 & 0 \\
0 & 0 & 0 \\
0 & 0 & 0
\end{array}\right)
$$$$
S_{21}=\left(\begin{array}{lll}
0 & 0 & 0 \\
1 & 0 & 0 \\
0 & 0 & 0
\end{array}\right)
$$$$
E_{1}=\left(\begin{array}{lll}
1 & 0 & 0 \\
0 & 0 & 0 \\
0 & 0 & 0
\end{array}\right)
$$$$
S_{12}=\left(\begin{array}{lll}
0 & 1 & 0 \\
0 & 0 & 0 \\
0 & 0 & 0
\end{array}\right)
$$$$
S_{21}=\left(\begin{array}{lll}
0 & 0 & 0 \\
0 & 0 & 0 \\
0 & 0 & 0
\end{array}\right)
$$

$E_{1}=\left(\begin{array}{lll}1 & 0 & 0 \\ 0 & 0 & 0 \\ 0 & 0 & 0\end{array}\right)$

$$
S_{12}=\left(\begin{array}{lll}
0 & 1 & 0 \\
0 & 0 & 0 \\
0 & 0 & 0
\end{array}\right)
$$$$
S_{21}=\left(\begin{array}{lll}
0 & 0 & 0 \\
0 & 0 & 0 \\
1 & 0 & 0
\end{array}\right)
$$

$$
E_{1}=\left(\begin{array}{lll}
1 & 0 & 0 \\
0 & 0 & 0 \\
0 & 0 & 0
\end{array}\right)
$$$$
S_{12}=\left(\begin{array}{lll}
0 & 1 & 0 \\
0 & 0 & 0 \\
0 & 0 & 0
\end{array}\right)
$$$$
S_{21}=\left(\begin{array}{lll}
0 & 0 & 0 \\
b & 0 & 0 \\
0 & 0 & 0
\end{array}\right)
$$

$$
E_{1}=\left(\begin{array}{lll}
1 & 0 & 0 \\
0 & 1 & 0 \\
0 & 0 & 0
\end{array}\right)
$$$$
S_{12}=\left(\begin{array}{lll}
0 & 0 & 0 \\
0 & 0 & 0 \\
0 & 0 & 0
\end{array}\right)
$$$$
S_{21}=\left(\begin{array}{lll}
0 & 0 & 0 \\
0 & 0 & 0 \\
0 & 0 & 0
\end{array}\right)
$$$$
E_{1}=\left(\begin{array}{lll}
1 & 0 & 0 \\
0 & 1 & 0 \\
0 & 0 & 0
\end{array}\right)
$$$$
S_{12}=\left(\begin{array}{lll}
0 & 0 & 0 \\
0 & 0 & 0 \\
0 & 0 & 0
\end{array}\right)
$$$$
S_{21}=\left(\begin{array}{lll}
0 & 0 & 0 \\
0 & 0 & 0 \\
0 & 1 & 0
\end{array}\right)
$$

$$
E_{1}=\left(\begin{array}{lll}
1 & 0 & 0 \\
0 & 1 & 0 \\
0 & 0 & 0
\end{array}\right)
$$$$
S_{12}=\left(\begin{array}{lll}
0 & 0 & 0 \\
0 & 0 & 1 \\
0 & 0 & 0
\end{array}\right)
$$$$
S_{21}=\left(\begin{array}{lll}
0 & 0 & 0 \\
0 & 0 & 0 \\
0 & 0 & 0
\end{array}\right)
$$$$
E_{1}=\left(\begin{array}{lll}
1 & 0 & 0 \\
0 & 1 & 0 \\
0 & 0 & 0
\end{array}\right)
$$$$
S_{12}=\left(\begin{array}{lll}
0 & 0 & 0 \\
0 & 0 & 1 \\
0 & 0 & 0
\end{array}\right)
$$$$
S_{21}=\left(\begin{array}{lll}
0 & 0 & 0 \\
0 & 0 & 0 \\
1 & 0 & 0
\end{array}\right)
$$

$E_{1}=\left(\begin{array}{lll}1 & 0 & 0 \\ 0 & 1 & 0 \\ 0 & 0 & 0\end{array}\right)$

$S_{12}=\left(\begin{array}{lll}0 & 0 & 0 \\ 0 & 0 & 1 \\ 0 & 0 & 0\end{array}\right)$

$S_{21}=\left(\begin{array}{lll}0 & 0 & 0 \\ 0 & 0 & 0 \\ 0 & c & 0\end{array}\right)$

for $c \in k^{*}$ 
We shall write $T_{3,1}-T_{3,6}, T_{3,7, b}, T_{3,8}-T_{3,11}$, and $T_{3,12, c}$ for the corresponding $S$-modules of dimension three. Notice that all $S$-modules of dimension three are extensions of simple $S$ modules of dimension one and two, so there are no simple $S$-modules of dimension $n=3$.

We remark that if $T$ is a simple $S$-module, then $\rho: S \rightarrow \operatorname{End}_{k}(T)$ is a surjective ring homomorphism. Hence it seems unlikely that there are any simple $S$-modules of dimension $n>3$.

Finally, we remark that the commutative deformation functor Def $_{M}: I \rightarrow$ Sets of the direct sum $M=M_{1} \oplus M_{2}$ has pro-representing hull $\left(H=k\left[\left[s_{12}, s_{21}\right]\right], M_{H}\right)$, and an algebraization $\left(S=k\left[s_{12}, s_{21}\right], M_{S}\right)$. It is not difficult to find the family $M_{S}$ in this case. In fact, for any point $(\alpha, \beta) \in \operatorname{Spec} S=\mathbf{A}_{k}^{2}$, the left $D$-module $M_{\alpha, \beta}=M_{S} \otimes_{S} S /\left(s_{12}-\alpha, s_{21}-\beta\right)$ is given by

$$
\begin{array}{ll}
M_{0,0} \cong M_{1} \oplus M_{2} & \\
M_{\alpha, 0} \cong D / D \cdot(\partial t) & \text { for } \alpha \neq 0 \\
M_{\alpha, \beta} \cong D / D \cdot(t \partial-\alpha \beta) & \text { for } \beta \neq 0
\end{array}
$$

We see that we obtain exactly the same isomorphism classes of left $D$-modules as commutative deformations of $M=M_{1} \oplus M_{2}$ as we obtained as noncommutative deformations of the family $\mathcal{M}=\left\{M_{1}, M_{2}\right\}$. However, the points of the pro-representing hull

$$
H=\left(\begin{array}{ll}
H_{11} & H_{12} \\
H_{21} & H_{22}
\end{array}\right) \cong\left(\begin{array}{cc}
k\left[\left[s_{12} s_{21}\right]\right] & \left\langle s_{12}\right\rangle \\
\left\langle s_{21}\right\rangle & k\left[\left[s_{21} s_{12}\right]\right]
\end{array}\right)
$$

of the noncommutative deformation functor $\operatorname{Def}_{\mathcal{M}}$ give a much better geometric picture of the local structure of the moduli space of left $D$-modules.

\section{References}

[1] Eivind Eriksen. An introduction to noncommutative deformations of modules. In Noncommutative algebra and geometry, volume 243 of Lect. Notes Pure Appl. Math., pages 90-125. Chapman \& Hall/CRC, Boca Raton, FL, 2006.

[2] Olav Arnfinn Laudal. Noncommutative deformations of modules. Homology Homotopy Appl., 4(2, part 2):357-396 (electronic), 2002. The Roos Festschrift volume, 2.

[3] Olav Arnfinn Laudal. Noncommutative algebraic geometry. In Proceedings of the International Conference on Algebraic Geometry and Singularities (Spanish) (Sevilla, 2001), volume 19, pages 509-580, 2003.

[4] Olav Arnfinn Laudal. The structure of $\operatorname{Simp}_{<\infty}(A)$ for finitely generated $k$-algebras $A$. In Computational commutative and non-commutative algebraic geometry, volume 196 of NATO Sci. Ser. III Comput. Syst. Sci., pages 3-43. IOS, Amsterdam, 2005. 